\title{
Rulers and sensors in Chlamydomonas
}

\section{cef}

the absolute flagella length may be translated into a posttranslational modification
Flagellum assembly and function in the green alga Chlamydomonas reinhardtii depend on complex mechanisms of sensing, both internally, to detect whether flagella are appropriately assembled, and externally, to modulate flagellar activity in response to environmental cues. Two reports this month advance our understanding of these processes. In Current Biology, Luo et al. show that the phosphorylation status of a flagellar regulatory protein is related to the absolute length of the flagella, and in Nature Cell Biology, Fujiu et al. identify the flagellar mechanoreceptor that allows $C$. reinhardtii to swim backwards on collision (the avoiding reaction).

C. reinhardtii has two apically localized flagella that it maintains at equal lengths $(11.6 \mu \mathrm{m} \pm 1.1 \mu \mathrm{m})$, but how it does this is not well understood. It had previously been shown that C. reinhardtii Aurora-like kinase (CALK) is phosphorylated in the absence of flagella and unphosphorylated in the presence of flagella. In the current study, Luo et al. investigated whether CALK phosphorylation is linked to flagellar length, using a $\mathrm{pH}$ shock to cause deflagellation and then following the synchronous reassembly of the flagella. They found that CALK is dephosphorylated when flagella are around half their usual length, rather than on the completion of flagellar assembly, as might have been expected. Next, they treated deflagellated cells with colchicine to stop flagellar assembly post-initiation. CALK in these flagella never became dephosphorylated, showing that the dephosphorylation is not related to the initiation of assembly itself or the time from the beginning of assembly, but is probably related to flagellar length. The authors then investigated whether CALK dephosphorylation is related to the absolute or relative length of the flagella. They studied two $C$. reinhardtii mutants that produce long $(20 \mu \mathrm{m})$ and short $(6-8 \mu \mathrm{m})$ flagella, respectively. In both of these mutants, CALK was dephosphorylated when flagella were $\sim 6 \mu \mathrm{m}$ in length; thus, CALK phosphorylation acts as a molecular measure of absolute flagellar length and is the first example showing that the absolute flagellar length may be translated into a post-translational modification, suggesting the presence of a length-sensing system.
Turning to flagellar activity, Fujiu et al. used a candidate gene approach and RNA interference to identify the receptor TRP11 as the mechanoreceptor responsible for the avoiding reaction of C. reinhardtii. This reaction allows the algae to swim backwards on collision and is mediated by a change in flagellar beating pattern, which is caused by the generation of a mechanoreceptor potential. Immunostaining showed that TRP11 is located in the proximal region of the flagellum. The authors suggest that this localization in the less motile part of the flagellum avoids activation by flagellar beating.

Flagella are widely studied structures, both those of $C$. reinhardtii and the related structures known as cilia in higher eukaryotes. These two studies, which address unanswered questions in the field, provide insights into how post-translational modification and localization of regulatory factors are important for flagellar function.

Joanna E. Huddleston

ORIGINAL RESEARCH PAPERS Luo, M. et al. The phosphorylation state of an Aurora-like kinase marks the length of growing flagella in Chlamydomonas. Curr. Biol. 21, 586-591 (2011)| Fujiu, K. et al. Mechanoreception in motile flagella of Chlamydomonas. Nature Cell Biol. 13, 630-632 (2011)

FURTHER READING Ginger, M. L., Portman, N., \& McKean, P. G. Swimming with protists: perception, motility and flagellum assembly. Nature Rev. Microbiol. 6, 838-850 (2008) 